\title{
Simple Electrical Modulation Scheme for Laser Feedback Imaging
}

\author{
Karl Bertling, Member, IEEE, Thomas Taimre, Associate Member, IEEE, Gary Agnew, Member, IEEE, \\ Yah Leng Lim, Member, IEEE, Paul Dean, Dragan Indjin, Sven Höfling, Senior Member, IEEE, \\ Robert Weih, Martin Kamp, Michael von Edlinger, Johannes Koeth, Member, IEEE, \\ and Aleksandar D. Rakić, Senior Member, IEEE
}

\begin{abstract}
In this paper, we demonstrate a simple square-wave electrical modulation scheme for imaging with laser feedback interferometry (LFI). Distinct advantages of this scheme include: 1) the straightforward creation of the modulating signal, even for high-current lasers and 2) its natural suitability for lock-in detection. We compare this simple scheme against two established imaging modalities for LFI: 1) mechanical modulation using an optical chopper and 2) the swept-frequency feedback interferometry approach. The proposed scheme lends itself to high-frequency modulation, which paves the way for high framerate LFI imaging with no motion artefacts using off-the-shelf equipment.
\end{abstract} lasers.

Index Terms - Laser feedback, interferometry, semiconductor

\section{INTRODUCTION}

$\mathbf{E}$ XPERIMENTALLY simple schemes for high frame-rate imaging using laser feedback interferometry (LFI) are of fundamental interest for many applications [1]-[3].

Manuscript received February 16, 2015; revised July 7, 2015 and November 23, 2015; accepted November 29, 2015. Date of publication December 9, 2015; date of current version February 10, 2016. This work was supported in part by the State of Bavaria, in part by the European Cooperation in Science and Technology under Grant BM1205, and in part by the Australian Research Council's Discovery Projects under Grant DP 120 103703. The work of Y. L. Lim was supported by the Queensland Government's through the Smart Futures Fellowships Programme. The work of P. Dean was supported by the Engineering and Physical Sciences Research Council, U.K. The work of S. Höfling was supported by the Royal Society and the Wolfson Foundation. The associate editor coordinating the review of this paper and approving it for publication was Dr. Shoushun Chen.

K. Bertling, G. Agnew, Y. L. Lim, and A. D. Rakić are with the School of Information Technology and Electrical Engineering, University of Queensland, Brisbane, QLD 4072, Australia (e-mail: bertling@itee.uq.edu.au; gary.agnew@uq.net.au; ylim@itee.uq.edu.au; rakic@itee.uq.edu.au).

T. Taimre is with the School of Mathematics and Physics, The University of Queensland, Brisbane, QLD 4072, Australia (e-mail: t.taimre@uq.edu.au).

P. Dean and D. Indjin are with the School of Electronic and Electrical Engineering, University of Leeds, Leeds LS2 9JT, U.K. (e-mail: p.dean@leeds.ac.uk; d.indjin@leeds.ac.uk).

S. Höfling is with the School of Physics and Astronomy, University of St Andrews, St Andrews KY16 9SS, U.K., also with Technische Physik, Physikalisches Institut, Universität Würzburg, Würzburg D-97074, Germany, and also with the Wilhelm Conrad Röntgen Research Center for Complex Material Systems, Universität Würzburg, Würzburg D-97074, Germany (e-mail: sven.hoefling@physik.uni-wuerzburg.de).

R. Weih and M. Kamp are with Technische Physik, Physikalisches Institut, Universität Würzburg, Würzburg D-97074, Germany, and also the Wilhelm Conrad Röntgen Research Center for Complex Material Systems, Universität Würzburg, Würzburg D-97074, Germany (e-mail: robert.weih@ physik.uni-wuerzburg.de; martin.kamp@physik.uni-wuerzburg.de).

M. von Edlinger and J. Koeth are with nanoplus GmbH, Gerbrunn 97218, Germany (e-mail: michael.v.edlinger@nanoplus.com; koeth@nanoplus.com).

Digital Object Identifier 10.1109/JSEN.2015.2507184
Most of the LFI imaging techniques (which utilise the self-mixing effect) proposed to date are limited in achievable modulation frequency (and consequently the imaging frame-rate) by the mechanical nature of the modulation scheme or the complexity of the electrical modulation used. To create the LFI signal some of these methods use an optical chopper [4], [5], or require longitudinal displacement of the object being imaged [6]. Mechanical modulation can also be achieved by using microelectromechanical devices, thus significantly decreasing the size of the complete system [7], [8]. Alternatively they require involved electrical or electro-optical modulation and detection schemes including frequency shifting of the laser beam by acousto-optic deflectors [9]-[11] or swept-frequency feedback interferometry with directly modulated lasers [12]-[16]. The LFI signal can also be detected without additional modulation of the laser carrier, by measuring the extremely small variations in the DC signal while scanning [17], [18]. However, the LFI signal can easily be obscured by fluctuations (a consequence of motion artefacts) when the scanning is rapid.

To overcome these limitations, we propose an ultimately simple electrical modulation scheme for LFI imaging using square-wave modulation of the laser current. Three sets of experiments were performed on the same imaging target to compare this simple modulation scheme against two established LFI imaging methods. Additional displacement experiments and simulations were carried out to elucidate on the nature of the observed interference fringes. In all cases, experiments were carried out using a mid-infrared (MIR) interband cascade laser (ICL) [19], [20]. The extremely simple nature of the square-wave laser current modulation scheme paves the way for high frame-rate imaging at high laser driving currents, from which MIR and THz QCL imaging applications may benefit significantly [21]-[23].

\section{EXPERIMENTAl Setup}

The experimental setup of the LFI system can be seen in Fig. 1. The MIR distributed feedback (DFB) ICL $(\lambda=3.57 \mu \mathrm{m})$ used in this study was a design adapted from [24] and operating characteristics (light-current, currentvoltage curves, and the emission spectrum) were described in detail in [5]. The device was designed specifically for optical spectroscopy of organic molecules and exhibits high level of phase stability and narrow emission linewidth. The package 


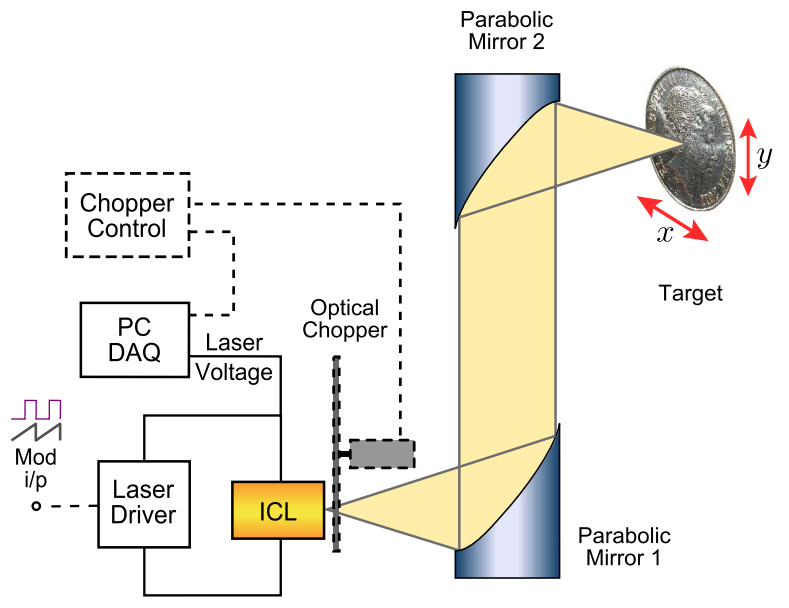

Fig. 1. Schematic of the experimental apparatus used.

used enables highly accurate temperature control making this laser eminently suitable for comparing different modulation schemes proposed in this article.

The ICL was kept at a constant temperature of $20^{\circ} \mathrm{C}$ using a Peltier temperature controller mounted inside the laser package, and operated at a drive current of $65 \mathrm{~mA}$, approximately $1.3 I_{\text {th }}$ (current threshold $I_{\text {th }}=50 \mathrm{~mA}$ ) with an output power of about $1.4 \mathrm{~mW}$. The emitted radiation from the ICL was collimated using a 2 inch diameter, 2 inch focal length off-axis parabolic reflector and focused normally on the target using a second identical reflector (giving an optical path length of $341.6 \mathrm{~mm}$ ). The voltage signal across the laser terminals was ac-coupled into a $\times 1000$ gain differential amplifier and subsequently fed into a 16-bit PC-based data acquisition card synchronised with the chopper or signal generator.

The ICL was operated in three different modes: (1) mechanically modulated using an optical chopper; (2) electrically modulated with a square-wave current signal; and (3) electrically modulated with a saw-tooth current signal. Each measurement data point (spatial pixel) was obtained using 64 averaged timedomain waveforms. All experiments were carried out using a $1 \mathrm{kHz}$ modulation frequency. Imaging experiments were performed on an Australian 5-cent coin and in the subsequent displacement experiments an aluminium front surfaced mirror was used. In all experiments, targets were mounted on a threeaxis computer-controlled translation stage, allowing each to be displaced along the optical axis of the system $(z)$ or raster scanned in a plane perpendicular to the optical axis $(x-y)$.

\section{A. Mechanical Modulation Using Optical Chopper}

The ICL beam was modulated at $1 \mathrm{kHz}$ with an optical chopper placed just in front of the output aperture of the laser, thereby amplitude-modulating the optical feedback which the laser was experiencing. Two states are present: (1) the chopper blade is obstructing the beam - the laser is operating with virtually no feedback; and (2) the beam is transmitted between the blades and to the external target - the laser is operating with an external feedback level dictated by the target. These two states result in two distinct voltage levels across the ICL, giving rise to a square-wave LFI signal in the time domain. (a)
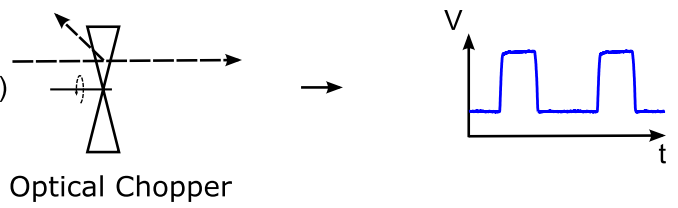

(b)

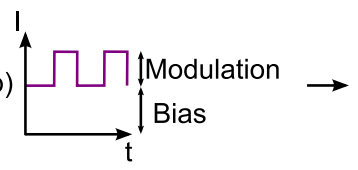

Square Wave Current Modulation

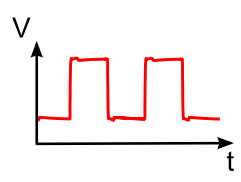

(c)

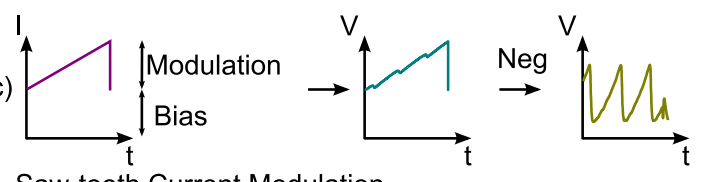

Saw-tooth Current Modulation

Fig. 2. Diagram of the three modulation schemes and their resulting LFI signals: (a) optical chopper; (b) square-wave current modulation; and (c) saw-tooth current modulation.

Figure 2(a) shows a representative voltage waveform measured across the laser terminals using this approach. This waveform clearly shows two states - the root-mean-square (RMS) of these waveforms (trimmed to central $90 \%$ of values) was used for image formation.

\section{B. Square-Wave Current Modulation}

A square-wave modulation of the laser current was applied with a frequency of $1 \mathrm{kHz}$ superimposed on the constant laser drive current with a modulation depth of $1 \mathrm{~mA}$. Square-wave modulation has a range of advantages in terms of ease of implementation and the availability of high-speed high-current off-the-shelf equipment that can be used to implement it. However, we would like to point out that a number of phenomena are involved in determining the voltage levels across the laser at the two current levels used. Firstly, as the laser sensitivity to feedback changes with bias current [4], [25] in particular in close to the lasing threshold, this type of modulation creates the LFI signal corresponding to two different bias points, in an alternating pattern [see Fig. 2(b)]. Secondly, due to current-induced frequency shift between these two states, the laser is essentially operating at two different frequencies corresponding to two different phase shifts accumulated in the external cavity, which the laser interferometer converts into two intensity levels with their corresponding voltages. One should keep in mind that the feedback-caused voltage variation is superimposed on the (much bigger) voltage change caused directly by the changes in laser driving current. All these phenomena contribute to the observed voltage signal, with their relative contribution depending on the laser type and the current levels used. The RMS value of the measured and trimmed voltage waveform was used for image formation.

\section{Saw-Tooth Current Modulation}

A saw-tooth modulation of the laser current was applied with a frequency of $1 \mathrm{kHz}$ and a modulation depth of $1 \mathrm{~mA}$. The ramp of the saw-tooth not only linearly modulates the 


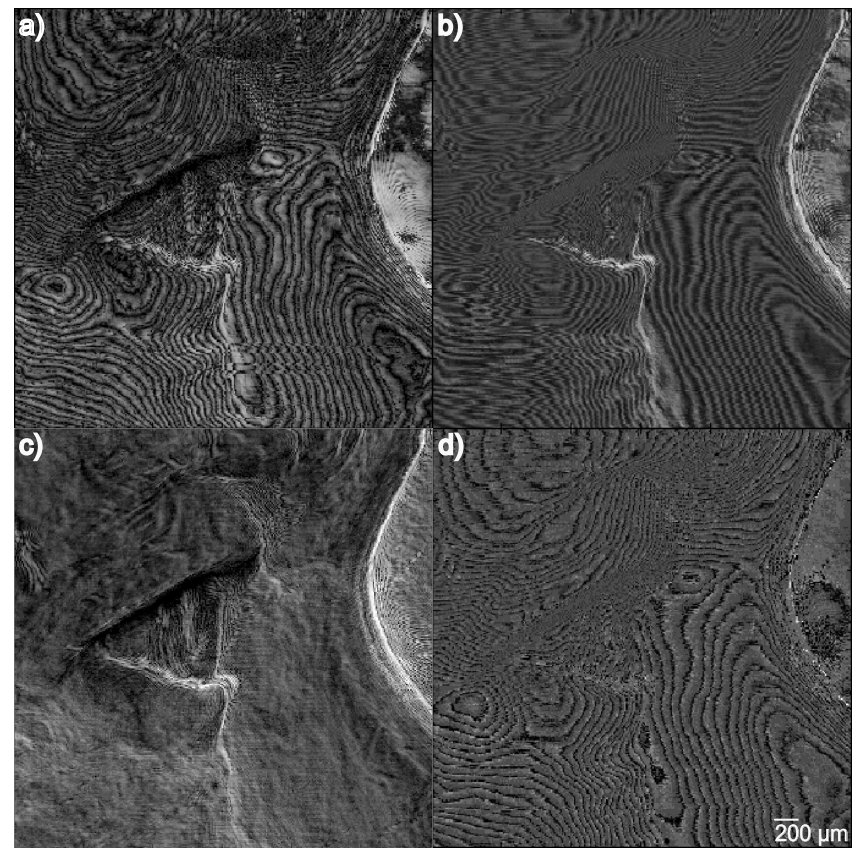

Fig. 3. Images of a small region on an Australian 5-cent coin, using the three modulation schemes. a) Optical chopper (RMS). b) Square-wave laser current modulation (RMS). c) Saw-tooth current modulation amplitude-like (peak-to-peak). d) Saw-tooth current modulation phase-like (peak position).

output power of the laser but also induces a linear sweep of the laser frequency. Figure 2(c) shows a typical waveform from this modulation scheme as measured across the laser terminals. The information-bearing portion of the measured waveform is the signal riding on the saw-tooth - Fig. 2(c) also shows the same waveform after the ramp has been removed ('negatised') allowing the interferometric fringes in the waveform to be clearly seen. This waveform contains information about target reflectivity and the phase-shift on reflection [15]. Therefore, two images can be obtained concurrently; one being representative of the strength of reflection at any given point, and the other corresponding to the phase-shift on reflection at the same point. The first of these images was obtained by extracting the peak-to-peak voltage of the negatised and trimmed signal, while the position of the last peak relative to the modulation period was used to obtain the phase image.

\section{RESULTS AND DISCUSSION}

\section{A. Imaging With Laser Feedback Interferometry}

The three modulation schemes were used to image the same $1.5 \times 1.5 \mathrm{~mm}^{2}$ region (with $5 \mu \mathrm{m}$ pitch) on the obverse of an Australian 5-cent coin. The common current bias point for the three schemes was $65 \mathrm{~mA}$, near to where the maximum LFI signal was observed using the mechanical modulation scheme. Figure 3 shows the results of imaging for the target with each of the three schemes. In each of the four images, the three dimensional nature of the target is clearly visible. Figures 3(a), 3(b), and 3(d) show interferometric fringes arising from the changing surface profile of the target on the scale of similar order as the laser wavelength. In Fig. 3(c) these interferometric fringes are conspicuously absent due to
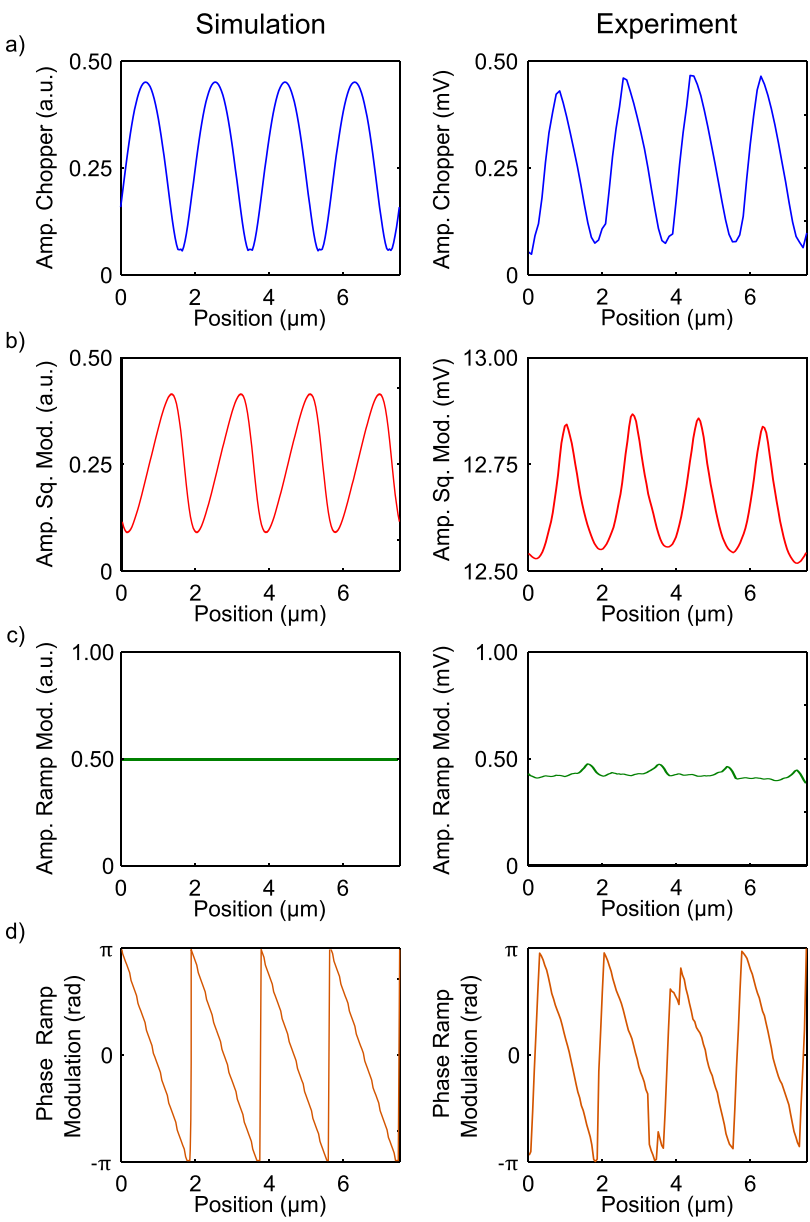

Fig. 4. Signal strength each modulation scheme over displacement: simulated (left) and experimental (right, biased at $65 \mathrm{~mA}$ ) results for the three modulation schemes. a) Optical chopper. b) Square-wave current modulation (1 mA modulation depth). c) Saw-tooth current modulation (amplitude-like). d) Saw-tooth current modulation (phase-like).

the amplitude-like nature of this representation; the phase information has been decoupled and is shown in Fig. 3(d).

\section{B. Displacement Characteristics of the Three Schemes}

To illuminate the link between the observed fringes and the operation of the laser feedback interferometer, we carried out an additional set of experiments and simulations. In these experiments, the target (a planar front surface aluminium mirror) was displaced longitudinally to reveal the effect on the LFI signal. The displacement used - four half-wavelengths $(7.148 \mu \mathrm{m})$ - corresponds to a change in transmission phase accumulated in the external cavity of $4 \pi$, and results in interferograms with four peaks. The right-hand column of Fig. 4 shows the experimentally measured dependence of the LFI signal on position, where the corresponding simulations appear in the left-hand column of the same figure. The simulation model used is based on the well-known excess phase equation governing self-mixing phenomena in lasers in steady-state and has been described in detail in [26]. Modulation from the optical chopper [Fig. 4(a)] was incorporated into simulation by periodically switching between a low reflectivity target when the optical chopper blade blocks the beam and a target 
with high reflectivity when the beam passes between chopper blades to the mirror at two distances from the laser. This was implemented by changing the value of the feedback parameter $C$ (a commonly used quantity for characterizing feedback levels [27]) between a low $(C=0.001)$ and a high $(C=1)$ value, and using two distances given by the geometry of the experimental setup. A clear match between simulation and experiment was observed.

Square-wave current modulation was captured in simulation by periodically switching between two different operating frequencies at two different voltage levels (corresponding to the two different driving currents). It is interesting to note that the observed change in RMS of the simulated signal with displacement was modelled solely through the difference in the transmission phase accumulated in the external cavity at each of the two operating frequencies and the change in laser sensitivity to feedback with the driving current was ignored.

The effect of saw-tooth current modulation on the negatised LFI signal was included in simulation as a linear frequency chirp over the modulation period as dictated by the frequency modulation coefficient of the laser. For each position of the target, this frequency chirp results in two or more ripples (peaks) in the LFI signal waveform - corresponding to a change in transmission phase of greater than $4 \pi$. The position of these peaks relative to the modulation waveform was determined by the length of the external cavity. As the target was linearly displaced, there was a corresponding linear change in the transmission phase, which was clearly captured [Fig. 4(d)] both in experiment and simulation.

On the other hand, the peak-to-peak amplitude of this saw-tooth current modulation signal should not change with displacement, as properly reflected in our simulation [Fig. 4(c)]. The experimental signal displays traces of the phase information seeping into the peak-to-peak amplitude.

This explains the presence of the interference fringes in Figs 3(a), 3(b), and 3(d) and their (almost complete) absence in Fig. 3(c).

\section{Discussion}

Both experiment and simulation show that the effects leading to the image formation were the change in the external cavity length and the change in the effective reflectivity [in this case the reflectivity of the target (5-cent coin) is changing due to change in angle and roughness at different points where the beam interrogates the surface]. The displacement experiment, where the reflectivity of the target was kept constant, clearly separates the two effects. For the first two modulation schemes [see Figs. 4(a) and (b)] the amplitude of the LFI signal depends on the displacement of the target; in the third case, due to the FM nature of the modulation scheme, it is the phase, not the amplitude of the LFI signal that contains the information about the displacement of the target. In all three cases, interference fringes (caused by the phase wrapping) are observable in the obtained images [see Figs. 4(c) and (d)]. The change in the effective reflectivity of the target additionally modulates the strength of the signal, and was clearly separated from the phase information in Fig. 3(c).
Each of the three modulation schemes has some comparative advantages. The mechanical modulation through the optical chopper produces simple output signal - lending itself to straightforward lock-in detection - and results in the greatest contrast with displacement, but has the modulation frequency limited by mechanical considerations. At high speeds it also introduces spurious mechanical vibrations and periodic changes in the refractive index of the air that will be detected by the system [28].

The square-wave current modulation can be implemented at high frequencies for high-current lasers (for example $\mathrm{THz}$ QCLs) - limited only by the characteristics of the laser being used - whilst retaining natural suitability for lock-in detection. Moreover, as no mechanical modulation is required, this scheme is more compact, requires fewer elements, and avoids any problems resulting from mechanical vibrations.

Finally, the saw-tooth current modulation results in two distinct concurrently captured images - each bearing complementary information about the target. However, the generation and detection of the modulating current waveforms and resulting voltage signals is considerably more involved, especially for high current lasers.

\section{CONCLUSION}

We have proposed a very simple square-wave electrical modulation scheme for imaging with LFI. The simplicity of the modulating current waveform lends itself to high frequency modulation with high current lasers and detection using offthe-shelf equipment. The proposed scheme compares well with established LFI imaging modalities.

\section{REFERENCES}

[1] G. Giuliani, M. Norgia, S. Donati, and T. Bosch, "Laser diode selfmixing technique for sensing applications," J. Opt. A, Pure Appl. Opt., vol. 4, no. 6, pp. S283-S294, 2002.

[2] S. Donati, "Developing self-mixing interferometry for instrumentation and measurements," Laser Photon. Rev., vol. 6, no. 3, pp. 393-417, 2012.

[3] T. Taimre, M. Nikolić, K. Bertling, Y. L. Lim, T. Bosch, and A. D. Rakić, "Laser feedback interferometry: A tutorial on the self-mixing effect for coherent sensing," Adv. Opt. Photon., vol. 7, no. 3, pp. 570-631, 2015.

[4] P. Dean et al., "Terahertz imaging through self-mixing in a quantum cascade laser," Opt. Lett., vol. 36, no. 13, pp. 2587-2589, 2011.

[5] K. Bertling et al., "Demonstration of the self-mixing effect in interband cascade lasers," Appl. Phys. Lett., vol. 103, no. 23, p. 231107 , 2013.

[6] P. Dean et al., "Coherent three-dimensional terahertz imaging through self-mixing in a quantum cascade laser," Appl. Phys. Lett., vol. 103, no. 18, p. $181112,2013$.

[7] V. Annovazzi-Lodi, S. Merlo, and M. Norgia, "Measurements on a micromachined silicon gyroscope by feedback interferometry," IEEE/ASME Trans. Mechatronics, vol. 6, no. 1, pp. 1-6, Mar. 2001.

[8] V. Annovazzi-Lodi, S. Merlo, and M. Norgia, "Characterization of silicon microstructures by feedback interferometry," J. Opt. A, Pure Appl. Opt., vol. 4, no. 6, pp. S311-S317, 2002.

[9] R. Day, E. Lacot, F. Stoeckel, and B. Berge, "Three-dimensional sensing based on a dynamically focused laser optical feedback imaging technique," Appl. Opt., vol. 40, no. 12, pp. 1921-1924, 2001.

[10] O. Jacquin, S. Heidmann, E. Lacot, and O. Hugon, "Self-aligned setup for laser optical feedback imaging insensitive to parasitic optical feedback," Appl. Opt., vol. 48, no. 1, pp. 64-68, Jan. 2009.

[11] W. Glastre, E. Lacot, O. Jacquin, O. Hugon, and H. G. de Chatellus, "Sensitivity of synthetic aperture laser optical feedback imaging," J. Opt. Soc. Amer. A, vol. 29, no. 4, pp. 476-485, 2012. 
[12] S. Shinohara, H. Yoshida, H. Ikeda, K. Nishide, and M. Sumi, "Compact and high-precision range finder with wide dynamic range and its application," IEEE Trans. Instrum. Meas., vol. 41, no. 1, pp. 40-44, Feb. 1992

[13] T. Bosch, N. Servagent, R. Chellali, and M. Lescure, "Three-dimensional object construction using a self-mixing type scanning laser range finder," IEEE Trans. Instrum. Meas., vol. 47, no. 5, pp. 1326-1329, Oct. 1998

[14] E. Gagnon and J.-F. Rivest, "Laser range imaging using the self-mixing effect in a laser diode," IEEE Trans. Instrum. Meas., vol. 48, no. 3, pp. 693-699, Jun. 1999.

[15] A. D. Rakić et al., "Swept-frequency feedback interferometry using terahertz frequency QCLs: A method for imaging and materials analysis," Opt. Exp., vol. 21, no. 19, pp. 22194-22205, 2013.

[16] J. Keeley et al., "Three-dimensional terahertz imaging using sweptfrequency feedback interferometry with a quantum cascade laser," Opt. Lett., vol. 40, no. 6, pp. 994-997, 2015.

[17] R. Juškaitis, N. P. Rea, and T. Wilson, "Semiconductor laser confocal microscopy," Appl. Opt., vol. 33, no. 4, pp. 578-584, 1994.

[18] M. Wang and G. Lai, "Self-mixing microscopic interferometer for the measurement of microprofile," Opt. Commun., vol. 238, nos. 4-6, pp. 237-244, 2004.

[19] R. Weih, M. Kamp, and S. Höfling, "Interband cascade lasers with room temperature threshold current densities below $100 \mathrm{~A} / \mathrm{cm}^{2}$," Appl. Phys. Lett., vol. 102, no. 23, p. 231123, 2013.

[20] I. Vurgaftman et al., "Interband cascade lasers," J. Phys. D, Appl. Phys., vol. 48 , no. 12, p. 123001, 2015.

[21] F. P. Mezzapesa et al., "Continuous-wave reflection imaging using optical feedback interferometry in terahertz and mid-infrared quantum cascade lasers," IEEE Trans. Terahertz Sci. Technol., vol. 4, no. 5, pp. 631-633, Sep. 2014.

[22] Y. L. Lim et al., "High-contrast coherent terahertz imaging of porcine tissue via swept-frequency feedback interferometry," Biomed. Opt. Exp. vol. 5, no. 11, pp. 3981-3989, Nov. 2014.

[23] P. Dean et al., "Terahertz imaging using quantum cascade lasersA review of systems and applications," J. Phys. D, Appl. Phys., vol. 47, no. 37 , p. 374008,2014

[24] I. Vurgaftman et al., "Rebalancing of internally generated carriers for mid-infrared interband cascade lasers with very low power consumption," Nature Commun., vol. 2, p. 585, Dec. 2011.

[25] K. B. Rochford and A. H. Rose, "Simultaneous laser-diode emission and detection for fiber-optic sensor applications," Opt. Lett., vol. 20, no. 20, pp. 2105-2107, 1995

[26] R. Kliese et al., "Solving self-mixing equations for arbitrary feedback levels: A concise algorithm," Appl. Opt., vol. 53, no. 17, pp. 3723-3736, 2014.

[27] K. Petermann, Laser Diode Modulation and Noise. Boston, MA, USA: Kluwer, 1991

[28] K. Bertling et al., "Imaging of acoustic fields using optical feedback interferometry," Opt. Exp., vol. 22, no. 24, pp. 30346-30356, 2014

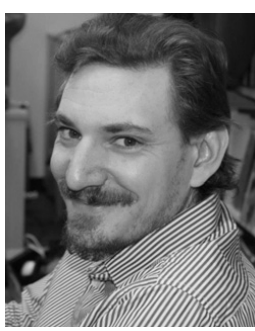

Karl Bertling (S'06-M'12) received the B.E. degree in electrical engineering, the B.Sc. degree in physics, the M.Phil. degree in electrical engineering, and the $\mathrm{Ph} . \mathrm{D}$. degree in electrical engineering from The University of Queensland, in 2003, 2006, and 2012 , respectively. He has contributed to the body of knowledge for this technique, in visible, nearIR, mid-IR, and terahertz semiconductor lasers. His current research interest are imaging and sensing via laser feedback interferometry (utilizing the selfmixing effect).

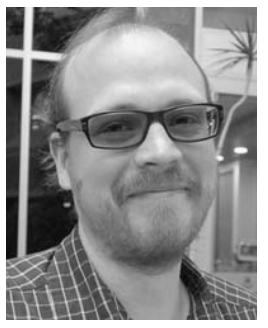

Thomas Taimre received the B.Sc. degree in mathematics and statistics, the B.Sc. (Hons.) degree in statistics, and the Ph.D. degree in mathematics from The University of Queensland, Australia, in 2003, 2004, and 2009, respectively. He is currently a Lecturer of Mathematics and Statistics with The University of Queensland. He has coauthored the book entitled Handbook of Monte Carlo Methods, which provides a hands-on guide to the theory, algorithms, techniques, and applications of Monte Carlo methods. His current research is at the interface of probability theory, computer simulation, and mathematical optimization with biological and other scientific, engineering, and finance disciplines, including within laser feedback interferometry.

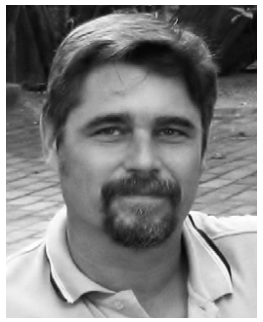

Gary Agnew (M'93) received the B.Sc. and M.Sc. degrees in electrical engineering from the University of the Witwatersrand, Johannesburg, South Africa, in 1985 and 1990, respectively. He is currently pursuing the Ph.D. degree with The University of Queensland, Australia. He has held a variety of research positions in the instrumentation industry, working on microwave, photonic, and nucleonic sensor technology. His research interests include modeling terahertz quantum cascade lasers.

Yah Leng Lim received the B.Eng. and Ph.D. degrees in electrical engineering from The University of Queensland, Brisbane, Australia, in 2001 and 2011, respectively. From 2002 to 2005, he joined Philips Optical Storage, Singapore, as an R\&D Engineer, working on the integration of optical and sensor technologies in optical storage systems. He currently holds a Smart Futures Fellowship funded by the Queensland Government, focusing on the development of LFI imaging systems for the early detection of skin cancers.

Paul Dean received the M.Phys. (Hons.) degree in physics and the Ph.D. degree in laser physics from the University of Manchester, Manchester, U.K. 2001 and 2005, respectively. In 2005, he was appointed as a Post-Doctoral Research Associate with the Institute of Microwaves and Photonics, School of Electronic and Electrical Engineering, University of Leeds, Leeds, U.K. His current research interests include terahertz optoelectronics, quantum cascade lasers, and terahertz imaging techniques. He received a fellowship from the Engineering and Physical Sciences Research Council (U.K.) in 2011.

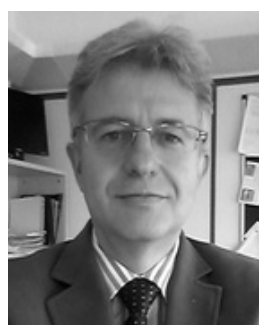

Dragan Indjin was born in Zemun, Yugoslavia, in 1963. He received the B.S., M.S., and $\mathrm{Ph} . \mathrm{D}$. degrees in electrical engineering from the University of Belgrade, Belgrade, Yugoslavia, in 1988, 1993, and 1996, respectively.

He joined the Faculty of Electrical Engineering University of Belgrade, in 1989, where he later became an Associate Professor. Since 2001, he has been with the Institute of Microwaves and Photonics, School of Electronic and Electrical Engineering, University of Leeds, Leeds, U.K., where he is currently a Reader (Associate Professor) in Optoelectronics and Nanoscale Electronics. He is currently focused on applications of quantum-cascade lasers and interband cascade lasers for sensing and imaging applications. His research interests include the electronic structures, optical and transport properties, optimization and design of quantum wells, superlattices, quantumcascade lasers, and quantum-well infrared photodetectors from near- to farinfrared and terahertz spectral ranges.

Dr. Indjin was a recipient of the prestigious Academic Fellowship from the Institute of Microwaves and Photonics, School of Electronic and Electrical Engineering, University of Leeds, in 2005 . He is currently a Coordinator of major international projects on infrared and terahertz imaging and sensing for medical and security applications.

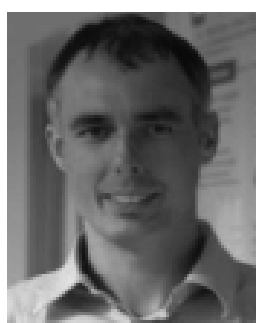

Sven Höfling (M'04-SM'13) was born in 1976. He received the Diploma degree in applied physics from the University of Applied Science, Coburg, and the Ph.D. degree from the Universität Würzburg, Germany. He was with the Fraunhofer Institute of Applied Solid-State Physics, Freiburg, Germany, from 2001 to 2002, working on blue and white light emitting diodes. In 2003, he joined the Universität Würzburg for his Ph.D. work on single mode emitting GaAs/AlGaAs quantum cascade lasers. From 2006 to 2013, he was the Head of the Optoelectronic Materials and Devices Group, Technische Physik, Universität Würzburg. He is currently a Professor of Physics with the Universität Würzburg, and the University of St Andrews, U.K.

His research interests include the design, fabrication, and characterization of low-dimensional electronic and photonic nanostructures, including quantum wells and quantum dots, organic semiconductors, high-quality factor microcavities, photonic crystal devices, semiconductor lasers, organic optoelectronics, and transition metal oxides.

Dr. Höfling is a member of the IEEE Photonics Society, the German Physical Society, the European Physical Society, the Institute of Physics, and the Optical Society. 
Robert Weih was born in 1985. He received the Diploma degree from the Universität Würzburg in 2011. He was working on mid infrared interband cascade lasers as a Ph.D. student with the Chair of Technische Physik, Universität Würzburg. His research interests include the growth and characterization of III-V semiconductors and the development of semiconductor devices, such as lasers and detectors. He is member of the German Physical Society.

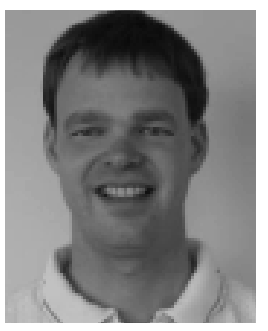

Martin Kamp received the master's degree from Stony Brook University, Stony Brook, NY, USA in 1995, and the Ph.D. degree from the Universität Würzburg, Würzburg, Germany, in 2003, for his work on laterally coupled distributed feedback lasers. He joined the Department of Technische Physik in 1996. From 2010 to 2015, he was the Interims Chair of the Department of Technische Physik. Since then, he is responsible for the nanofabrication and nanophotonic research with the Department of Technische Physik and the Gottfried-Landwehr Laboratory for Nanotechnology, Universität Würzburg. $\mathrm{He}$ has authored or co-authored over 250 papers relating to semiconductor nanostructures, opto- and nanoelectronic devices, and semiconductor spectroscopy.

His current research interests include low-dimensional electronic and photonic semiconductor structures and the development of semiconductorbased optical quantum information processing and interband cascade lasers.

Dr. Kamp is a member of the German Physical Society.

Michael von Edlinger was born in Bamberg, Germany, in 1982. He received the degree from the University of Würzburg with a focus on low-emitting ceramics based on $\mathrm{TiO} 2$ in 2008 . He started to work on his Ph.D. thesis at nanoplus in 2009. The main topic of his thesis is the design and fabrication of monolithic and widely tunable interband cascade laser sources for trace gas detection.

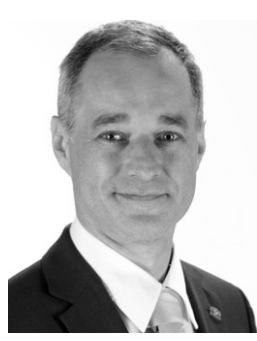

Johannes Koeth received the degree in physics from the Universität Würzburg with studies on molecular beam epitaxy (MBE) and optical properties of $\mathrm{GaN}$ in 1996. During his Ph.D., he worked on MBE of lasers in the $\mathrm{AlGaSb}$ system in the wavelength range between 1.5 and $2.0 \mu \mathrm{m}$. One of the main topics of his thesis was the investigation of GaSb-based emitters on $\mathrm{GaAs}$ substrates. He realized, e.g., the first $\mathrm{GaSb}$ edge emitting laser diodes based on GaAs and optically pumped $\mathrm{Ga}(\mathrm{Al}) \mathrm{Sb} / \mathrm{AlSb}$ VCSELs, both at $1.5 \mu \mathrm{m}$. In addition, he developed and characterized antimonide-based DFB lasers in the 2- $\mu \mathrm{m}$ range. In 1998, he participated to the creation of nanoplus nanosystems and technologies $\mathrm{GmbH}$ and has been the CEO of the company ever since.

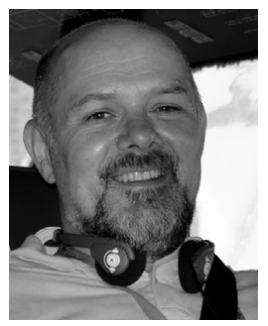

Aleksandar D. Rakić (M'93-SM'10) leads the Microwave, Photonics, and Communications Group with The University of Queensland, focusing on the development of technologies for sensing and imaging across the electromagnetic spectrum including microwave, terahertz wave, and optical systems. $\mathrm{He}$ is currently a Professor with the School of IT and Electrical Engineering, The University of Queensland. Over the past ten years, his group pioneered the development of several world's first laserfeedback interferometric sensors, including systems based on monolithic vertical-cavity surface-emitting laser arrays (VCSELs), blue-green lasers, terahertz quantum cascade lasers, and mid-infrared interband cascade lasers. His current research involves the development of sensing and imaging systems exploiting the $\mathrm{THz}$ spectrum for applications from security and defense to in vivo biomedical imaging. His other principal contributions relate to the design and characterization of surface-emitting optoelectronic devices (VCSELs and light emitting diodes). He was appointed as an Invited Professor with the University of Toulouse (2007-2012), and a Visiting Professor with the University of Leeds (2012). He served as the General Chair of the 2004 Conference on Optoelectronic and Microelectronic Materials and Devices, the Co-Chair of the Symposium on Molecular and Organic Electronics and Organic Displays within the 2006 International Conference on Nanoscience and Nanotechnology, and the Chair of the Symposium on Compound Semiconductor Materials and Devices within the 2008 International Conference on Electronic Materials and the IEEE AP/MTT Queensland Chapter. 